\title{
A FIXED POINT THEOREM FOR SEMIGROUPS OF MAPPINGS WITH A CONTRACTIVE ITERATE
}

\author{
V. M. SEHGAL AND J. W. THOMAS
}

Abstract. In a recent paper, Felix E. Browder discussed continuous self-mappings on a metric space, satisfying a functional inequality. Browder gave sufficient conditions such that the successive approximations of any point for such mappings converge to a unique fixed point. In the present paper, Browder's result is extended to a commutative semigroup of mappings and also to single mappings that are not necessarily continuous and satisfy a weaker form of the functional inequality.

1. It is the purpose of this paper to generalize the following result of Felix Browder [2], under the assumption that the set $M$ there is closed.

Theorem 1. Let $(X, d)$ be a complete metric space, $M$ a bounded subset of $X, T$ a mapping of $M$ into $M$. Suppose there exists a monotone nondecreasing function $\psi(r)$ for $r \geqq 0$ with $\psi$ continuous on the right, such that $\psi(r)<r$ for all $r>0$, while for $x, y \in M$,

$$
d(T(x), T(y)) \leqq \psi(d(x, y)) .
$$

Then, for each $x_{0} \in M, T^{n}\left(x_{0}\right) \rightarrow \xi \in X$, independent of $x_{0}$, and

$$
d\left(T^{n}\left(x_{0}\right), \xi\right) \leqq \psi^{n}\left(d_{0}\right),
$$

where $d_{0}$ is the diameter of $M, \psi^{n}$ is the $n$th iterate of $\psi$, and

$$
d_{n}=\psi^{n}\left(d_{0}\right) \rightarrow 0 \text { as } n \rightarrow \infty .
$$

It may be remarked that if $M$ is closed, then it follows that $\xi$ is the unique fixed point of $T$ in $M$, and (2) provides an estimate for $d\left(T^{n}\left(x_{0}\right), \xi\right)$. Boyd and Wong [1] have obtained a result similar to Theorem 1, without the estimate (2), where the domain of $T$ is unbounded.

2. Let $(X, d)$ be a complete metric space and $M \subseteq X$. Let $F$ be a commutative semigroup of self-mappings (not necessarily continuous) of $M$. The semigroup $F$ is pointwise contractive in $M$ if for each $x \in M$, there is an $f_{x} \in F$ such that

$$
d\left(f_{x}(y), f_{x}(x)\right) \leqq \psi(d(y, x))
$$

Received by the editors November 10, 1970.

AMS 1969 subject classifications. Primary 4785, 5485; Secondary 2654.

Key words and phrases. Pointwise contractive semigroup. 
for all $y \in M$, where $\psi$ is some real valued function defined on the nonnegative reals.

Theorem 2. Let $M$ be a closed subset of $X$ and $F$ a commutative semigroup of self-mappings of $M$, which is pointwise contractive in $M$ for some $\psi:[0, \infty) \rightarrow[0, \infty)$, where $\psi$ is nondecreasing, continuous on the right and satisfies $\psi(r)<r$ for all $r>0$. If for some $x_{0} \in M$,

$$
\sup \left\{d\left(f\left(x_{0}\right), x_{0}\right): f \in M\right\}<\infty,
$$

then, there exists a unique $\xi \in M$ such that $f(\xi)=\xi$ for each $f \in F$. Moreover, there is a sequence $\left\{g_{n}\right\} \subseteq F$ with $g_{n}(x) \rightarrow \xi$ for each $x \in M$.

Proof. Let $d_{0}=\sup \left\{d\left(f\left(x_{0}\right), x_{0}\right): f \in F\right\}$. Then, $\psi^{n}\left(d_{0}\right)$ is a nonincreasing sequence of nonnegative reals, and therefore, $\psi^{n}\left(d_{0}\right) \rightarrow r \geqq 0$. If $r>0$, then $\psi\left(\lim _{n} \psi^{n}\left(d_{0}\right)\right)<r$, that is, $r=\lim _{n} \psi^{n}\left(d_{0}\right)<r$. We conclude, therefore, that $\lim _{n} \psi^{n}\left(d_{0}\right)=0$.

Set $f_{0}=f_{x_{0}}$ and inductively $f_{n}=f_{x_{n}}$ where $x_{n+1}=f_{n}\left(x_{n}\right)$. Then, for a fixed integer $k \geqq 0$,

$$
\sup _{n \geqq k} d\left(x_{n+1}, x_{k+1}\right)=\sup _{n \geqq k} d\left(f_{n} \cdot f_{n-1} \cdots f_{k}\left(x_{k}\right), f_{k}\left(x_{k}\right)\right) .
$$

Set $h_{n}=f_{n} \cdot f_{n-1} \cdots f_{k+1}$. It follows that

$$
\begin{aligned}
\sup _{n \geqq \boldsymbol{k}} d\left(x_{n+1}, x_{k+1}\right) & =\sup _{n \geqq \boldsymbol{k}} d\left(f_{k}\left(h_{n}\left(x_{k}\right)\right), f_{k}\left(x_{k}\right)\right), \\
& \leqq \sup _{n \geqq \boldsymbol{k}} \psi\left(d\left(h_{n}\left(x_{k}\right), x_{k}\right)\right), \\
& \leqq \sup _{n \geqq \boldsymbol{k}} \psi^{k+1}\left(d\left(h_{n}\left(x_{0}\right), x_{0}\right)\right), \\
& \leqq \psi^{k+1}\left(d_{0}\right) \rightarrow 0 \quad \text { as } k \rightarrow \infty .
\end{aligned}
$$

The sequence $\left\{x_{n}\right\}$ is therefore, Cauchy. Let $x_{n} \rightarrow \xi \in M$. Then, by hypothesis, there is an $f_{\xi} \in F$ such that

$$
d\left(f_{\xi}\left(x_{n}\right), f_{\xi}(\xi)\right) \leqq \psi\left(d\left(x_{n}, \xi\right)\right) \rightarrow 0 \quad \text { as } n \rightarrow \infty .
$$

Thus, $f_{\xi}\left(x_{n}\right) \rightarrow f_{\xi}(\xi)$, and therefore $d\left(f_{\xi}(\xi), \xi\right)=\lim _{n} d\left(f_{\xi}\left(x_{n}\right), x_{n}\right)$. However,

$$
d\left(f_{\xi}\left(x_{n}\right), x_{n}\right) \leqq \psi\left(d\left(f_{\xi}\left(x_{n-1}\right), x_{n-1}\right)\right) \leqq \psi^{n}\left(d\left(f_{\xi}\left(x_{0}\right), x_{0}\right)\right) \leqq \psi^{n}\left(d_{0}\right) \rightarrow 0
$$

as $n \rightarrow \infty$. Hence $f_{\xi}(\xi)=\xi$. It follows from (3) that $\xi$ is the unique fixed point of $f_{\xi}$. Furthermore, by the commutativity of $F$, we have, for any $f \in F$,

$$
f(\xi)=f\left(f_{\xi}(\xi)\right)=f_{\xi}(f(\xi)),
$$

and therefore $f(\xi)=\xi$. 
Finally, for each nonnegative integer $n$, set $g_{n}=f_{n} \cdot f_{n-1} \cdots f_{0}$. Then $g_{n} \in F$. We show that $g_{n}(x) \rightarrow \xi$ for each $x \in M$. If $d(x)=d\left(x, x_{0}\right)$, then it follows that $\psi^{n}(d(x)) \rightarrow 0$. We have

$$
d\left(g_{n}(x), \xi\right) \leqq d\left(g_{n}(x), x_{n+1}\right)+d\left(x_{n+1}, \xi\right) .
$$

Since $x_{n} \rightarrow \xi$, it suffices to show that $d\left(g_{n}(x), x_{n+1}\right) \rightarrow 0$. However,

$$
\begin{aligned}
d\left(g_{n}(x), x_{n+1}\right) & =d\left(f_{n}\left(g_{n-1}(x)\right), f_{n}\left(x_{n}\right)\right), \\
& \leqq \psi\left(g_{n-1}(x), x_{n}\right), \\
& \leqq \psi^{n+1}\left(d\left(x, x_{0}\right)\right)=\psi^{n+1}(d(x)) \rightarrow 0 \quad \text { as } n \rightarrow \infty .
\end{aligned}
$$

Thus $d\left(g_{n}(x), x_{n+1}\right) \rightarrow 0$. This completes the proof.

If $M$ is a bounded subset of $X$, then since (4) holds for each $x_{0} \in M$, we have

Corollary 1. Let $M$ be a closed bounded subset of $X$, and $F$ a commutative semigroup of self-mappings of $M$ which is pointwise contractive in $M$ for some $\psi:[0, \infty) \rightarrow[0, \infty)$, where $\psi$ is nondecreasing, continuous on the right and satisfies $\psi(r)<r$ for $r>0$. Then there exist a sequence $\left\{g_{n}\right\} \subseteq F$ and a unique $\xi \in M$ such that $f(\xi)=\xi$ for all $f \in F$, and $g_{n}(x) \rightarrow \xi$ for each $x \in M$.

Corollary 2. Let $M$ be a closed bounded subset of $X$, and $f$ a selfmapping of $M$. If $f$ satisfies the condition: for each $x \in M$, there exists an integer $n(x) \geqq 1$ such that, for all $y \in M$,

$$
d\left(f^{n(x)}(y), f^{n(x)}(x)\right) \leqq \psi(d(y, x)),
$$

where $\psi:[0, \infty) \rightarrow[0, \infty)$ is nondecreasing, continuous on the right and satisfies $\psi(r)<r$ for $r>0$, then there is a unique $\xi \in M$ such that $f^{n}(x) \rightarrow \xi$ for each $x \in M$ and $f(\xi)=\xi$.

Proof. Since $F=\left\{f^{n}: n \geqq 0\right\}$ is a commutative semigroup which is pointwise contractive in $M$, the existence and uniqueness of $\xi \in M$ follow by Corollary 1 . We show that $f^{n}(x) \rightarrow \xi$ for each $x \in M$. Let $d_{0}$ be the diameter of the set $M$. Then if $n$ is sufficiently large, we have $n=r \cdot n(\xi)+s$, with $r>0$ and $0 \leqq s<n(\xi)$, and, therefore,

(6) $d\left(f^{n}(x), \xi\right)=d\left(f^{r \cdot n(\xi)+s}(x), f^{n(\xi)}(\xi)\right) \leqq \psi^{r}\left(d\left(f^{s}(x), \xi\right)\right) \leqq \psi^{r}\left(d_{0}\right)$.

Since $\psi^{n}\left(d_{0}\right) \rightarrow 0$, and $r \rightarrow \infty$ as $n \rightarrow \infty$, it follows therefore that $f^{n}(x) \rightarrow \xi$.

REMARK 1 . It should be noted that inequality (6) provides the analogue to Browder's estimate (2).

REMARK 2. For bounded metric spaces, Corollary 2 is an extension of a result of the first author [4] and also of a recent result of $L$. F. Guseman [2]. Note that Theorem 2 and its corollaries provide 
generalizations of Theorem 1 under the assumption that the set $M$ is closed.

\section{REFERENCES}

1. D. W. Boyd and J. S. W. Wong, On nonlinear contractions, Proc. Amer. Math Soc. 20 (1969), 458-464. MR 39 \#916.

2. Felix E. Browder, On the convergence of successive approximations for nonlinear functional equations, Nederl. Akad. Wetensch. Proc. Ser. A 71=Indag. Math. 30 (1968), 27-35. MR 37 \#5743.

3. L. F. Guseman, Jr., Fixed point theorems for mappings with a contractive iterate at a point, Proc. Amer. Math. Soc. 26 (1970), 615-618.

4. V. M. Sehgal, $A$ fixed point theorem for mappings with a contractive iterate, Proc. Amer. Math. Soc. 23 (1969), 631-634. MR 40 \#3531.

UNIVERSITY OF WYOMING, LARAMIE, WYOMING 82070 